\title{
Medial tibial stress syndrome: conservative treatment options
}

\author{
R. Michael Galbraith • Mark E. Lavallee
}

Published online: 7 October 2009

(C) The Author(s) 2009. This article is published with open access at Springerlink.com

\begin{abstract}
Medial tibial stress syndrome (MTSS), commonly known as "shin splints," is a frequent injury of the lower extremity and one of the most common causes of exertional leg pain in athletes (Willems T, Med Sci Sports Exerc 39(2):330-339, 2007; Korkola M, Amendola A, Phys Sportsmed 29(6):35-50, 2001; Hreljac A, Med Sci Sports Exerc 36(5):845-849, 2004). Although often not serious, it can be quite disabling and progress to more serious complications if not treated properly. Often, the cause of MTSS is multi-factorial and involves training errors and various biomechanical abnormalities. Few advances have been made in the treatment of MTSS over the last few decades. Current treatment options are mostly based on expert opinion and clinical experience. The purpose of this article is to review published literature regarding conservative treatment options for MTSS and provide recommendations for sports medicine clinicians for improved treatment and patient outcomes.
\end{abstract}

Keywords Medial tibial stress syndrome - Shin splints . Tibial stress injury $\cdot$ Lower extremity injuries

\section{Definition of medial tibial stress syndrome}

Medial tibial stress syndrome (MTSS) is an overuse injury or repetitive-stress injury of the shin area. Various stress reactions of the tibia and surrounding musculature occur

R. M. Galbraith $(\varangle) \cdot$ M. E. Lavallee

South Bend Primary Care Sports Medicine Fellowship,

111 W. Jefferson, Suite \# 100, South Bend, IN 46601, USA

e-mail: galbraithsfam@gmail.com

M. E. Lavallee

e-mail: mlavallee@memorialsb.org when the body is unable to heal properly in response to repetitive muscle contractions and tibial strain.

\section{Pathophysiology}

Many believe the main cause of MTSS involves underlying periostitis of the tibia due to tibial strain when under a load. However, new evidence indicates that a spectrum of tibial stress injuries is likely involved in MTSS, including tendinopathy, periostitis, periosteal remodeling, and stress reaction of the tibia [1-4]. Dysfunction of the tibialis posterior, tibialis anterior, and soleus muscles are also commonly implicated [1, 3, 4]. These various tibial stress injuries appear to be caused by alterations in tibial loading, as chronic, repetitive loads cause abnormal strain and bending of the tibia [1]. Although sometimes composed of different etiologies, MTSS and tibial stress fractures may be considered on a continuum of bone-stress reactions $[1,4]$.

\section{Materials and methods}

To identify appropriate literature, we searched multiple databases, journals, and sports medicine and orthopedic textbooks. Electronic databases included Medline Systematic Reviews, MD Consult, and the Cochrane Library. Primary care and sports medicine journals were reviewed, such as American Family Physician, Clinical Journal of Sports Medicine, British Journal of Sports Medicine, Clinics in Sports Medicine, Medicine and Science in Sports and Exercise, American Journal of Sports Medicine, and Physical Medicine and Rehabilitation Clinics of North America. 
We focused on papers that included treatments and rehabilitation of MTSS. We searched for the subject terms "shin splints," "medial tibial stress syndrome," "tibial stress injury," and "lower extremity injuries." We further narrowed the search with the terms "treatment" and "rehabilitation."

\section{Results}

Multiple review articles and case reports regarding treatment options for MTSS were identified. Most evidence was based on expert opinion and clinical experience, and there was a general paucity of randomized controlled trials (RCTs). Many studies focused on the etiology and risk factors for MTSS, rather than treatment. However, a recent systematic review of literature demonstrated serious methodological flaws in four RCTs [5]. It is apparent that few interventions and conservative treatment options for MTSS have undergone rigorous examination [5].

\section{Discussion}

\section{Clinical history}

The most common complaint of patients with MTSS is vague, diffuse pain of the lower extremity, along the middle-distal tibia associated with exertion $[1,6]$. In the early course of MTSS, pain is worse at the beginning of exercise and gradually subsides during training and within minutes of cessation of exercise. As the injury progresses, however, pain presents with less activity and may occur at rest.

A comprehensive history should be obtained to evaluate the athlete's weekly exercise routine and running mileage, intensity, pace, terrain, and footwear, with careful attention to recent changes in training regimens. Training errors appear to be the most common factors involved in MTSS, especially as athletes attempt to do "too much, too fast" [6-8]. Common training errors include a recent onset of increased activity, intensity, or duration [6-9]. Running on hard or uneven surfaces is also a common risk factor. Individuals with previous lower extremity injuries and running more than 20 miles per week are especially predisposed to overuse injuries of the lower extremity, including MTSS [10]. MTSS is most often found in runners, and also found in other ballistic sports, such as football, basketball, soccer, and dancing.

There appears to be a gender predilection as females are at a 1.5 to 3.5 times increased risk for progression to stress fractures $[10,11]$. Females have a higher incidence of diminished bone density and osteoporosis, as seen in the female athlete triad (osteoporosis, amenorrhea, and disordered eating) [10-12]. Clinicians should have a high index of suspicion of eating disorders and nutritional deficiencies, including hypocalcemia, in female athletes with abnormal menses and multiple stress fractures.

\section{Physical examination}

A combination of training errors and biomechanical abnormalities are key risk factors for developing MTSS [1, 6-8, 13]. Clinicians should complete a thorough musculoskeletal examination of the patient, with special focus on the lower extremity $[7,8,14]$. The medial ridge of the tibia (origin of the tibialis posterior and soleus muscles) is often tender to palpation, especially at the distal and middle tibial regions. The anterior tibia, however, is usually nontender. Neurovascular symptoms are usually absent [6].

MTSS is associated with biomechanical abnormalities of the lower extremity $[1,15,16]$. Physicians should carefully evaluate for possible knee abnormalities (especially genu varus or valgus), tibial torsion, femoral anteversion, foot arch abnormalities, or a leg-length discrepancy [1, 6, 9]. Ankle movements and subtalar motion should also be evaluated. Hyperpronation of the subtalar joint is one of the most common and well-documented risk factors for MTSS (see Fig. 1a, b) [1, 5-8, 12, 15, 17, 18].

Muscle imbalance and inflexibility, especially tightness of the triceps surae (gastrocnemius, soleus, and plantaris muscles), is commonly associated with MTSS [1, 7, 8].
Fig. 1 Hyperpronation of subtalar joint. a Medial, b anterior views. Courtesy: Courtney Sullivan
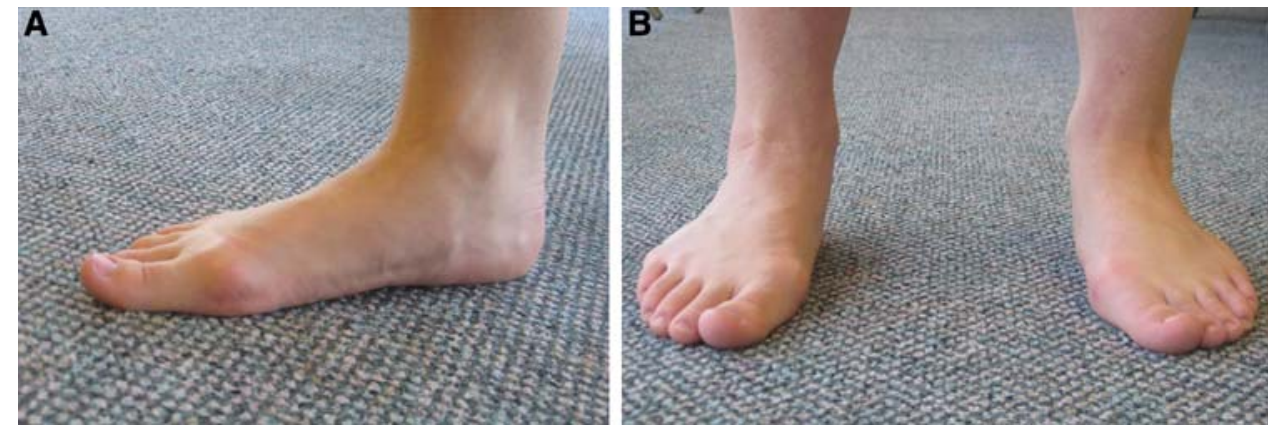
Athletes with muscle weakness of the triceps surae are more prone to muscle fatigue, leading to altered running mechanics, and strain on the tibia [1]. Clinicians should also examine for inflexibility and imbalance of the hamstring and quadriceps muscles.

Weakness of "core muscles" is an important risk factor for lower extremity injuries [16, 19-21]. Hip and pelvis muscle strength are an important link in maintaining control and proper mechanics between the "core" and lower extremity [16, 22]. Core and pelvic muscle stability may be assessed by evaluating patient's ability to maintain a controlled, level pelvis during a pelvic bridge from the supine position, or a standing single-leg knee bend (see Fig. 2).

Lower extremity injuries are also associated with structural abnormalities elsewhere in the kinetic chain, removed from the site of injury [8, 22-25]. Dysfunction of the spine, sacroiliac joint, and pelvis may contribute to injuries of the lower extremity [22, 25]. The physician should perform a standing postural examination to evaluate the entire kinetic chain, especially if the symptoms are recurrent [8, 22, 23]. Musculoskeletal abnormalities (somatic dysfunctions) can be identified by the mnemonic "TART": Tenderness, Asymmetry, Restricted motion, and Tissue texture abnormality [22]. These principles can be applied to the entire kinetic chain, including the muscles, soft tissues and bones of the spine, pelvis, and extremities. Several methods are used to detect leg-length discrepancies. Examining the patient in a supine position, while evaluating for equal medial malleoli, is simple and effective.

Examining patient's shoes may reveal generally wornout shoes or patterns consistent with a leg-length discrepancy or other biomechanical abnormalities. Abnormal gait patterns should be evaluated with the patient walking and

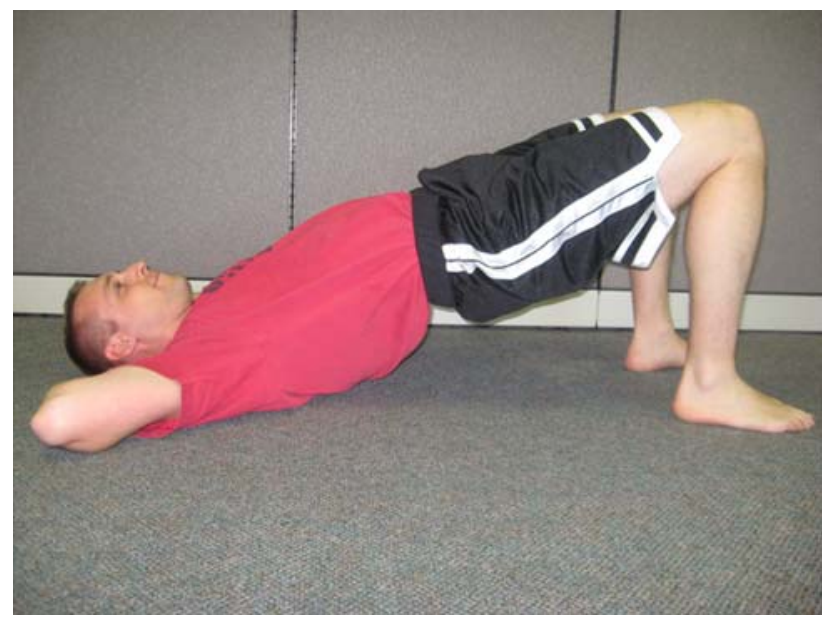

Fig. 2 Pelvic bridge exercise to strengthen gluteal core muscles. Courtesy: R. Michael Galbraith running, either in the office hallway or on a treadmill $[9,10]$.

Making the diagnosis

A thorough history and physical exam are usually sufficient to make the diagnosis of MTSS. Patients may require further imaging or work-up to rule out stress fractures or other pathology.

\section{Differential diagnosis}

The most common complication of MTSS is a stress fracture of the tibia, manifested by focal tenderness of the anterior tibia [10, 26]. Acute or chronic exertional compartment syndrome is one of the conditions most likely confused with MTSS. It should be considered especially when sensory or motor loss is present in association with exertional lower leg pain. Peripheral vascular disease is another common cause of exertional-leg pain in older and diabetic athletes. Physicians should also be aware of less common causes of lower extremity exertional-pain, including muscle tears, fascial defects, occult fracture, infection, neoplasms, effort-induced venous thrombosis, peroneal nerve entrapment, and popliteal artery entrapment syndrome. A thorough history and exam is usually sufficient to exclude other causes. Occasionally, further imaging, compartment pressure measurements, and vascular and nerve conduction studies are required to lead to the correct diagnosis [27].

\section{Imaging}

Imaging is usually not necessary for the diagnosis of MTSS. However, if the patient fails to improve with conservative management, plain film radiography may be considered. X-rays are usually negative within the first 2-3 weeks after the onset of injury [28]. Long-term radiographic changes of those with chronic MTSS with periosteal involvement may show periosteal exostoses. Those that progress from MTSS to stress fracture may develop a dreaded black line on radiograph, which indicates a more ominous sign [13].

A triple-phase bone scan demonstrates evidence of a stress fracture with a diffuse, longitudinal uptake along the posterior tibia, seen only on the delayed phase of the scan $[6,29]$. Bone scans have been the gold standard for diagnosing stress fractures in the past, but now are often replaced by magnetic resonance imaging (MRI).

MRI has many advantages over a bone scan and plane radiography. MRI is better able to identify other soft tissue injuries [4, 10, 29, 30]. MRI can show progression of injury in the tibia, starting with periosteal edema, progressive 
Table 1 Grading of tibial stress injuries by MRI [4]

\begin{tabular}{ll}
\hline Grade & Findings \\
\hline 1 & Periosteal edema \\
$2-3$ & Progressive periosteal and bone marrow edema \\
4 & Cortical stress fracture \\
\hline
\end{tabular}

marrow involvement, and finally cortical stress fracture (see Table 1) [4]. With better accuracy, MRI can grade tibial injuries according to extent of involvement. Grading tibial injuries may help clinicians make more accurate recommendations for rehabilitation, though no literature data exist about MRI findings and specific return-to-play guidelines [4].

Conservative treatment options

\section{Acute phase}

Rest, ice Most literature supports "rest" as the most important treatment in the acute phase of MTSS [1, 4, 6, 13, 30, 31]. For many athletes, however, prolonged rest from activity is not ideal, and other therapies are necessary to help the athlete return to activity quickly and safely. Patients may require "relative" rest and cessation of sport for prolonged periods of time (from 2 to 6 weeks), depending on the severity of their symptoms. NSAIDs and Acetaminophen are often used for analgesia. Cryotherapy is also commonly used in the acute period. Ice may be applied to the affected area directly after exercise for approximately $15-20 \mathrm{~min}$.

Therapy Physical therapy modalities, such as ultrasound, whirlpool baths, phonophoresis, augmented soft tissue mobilization, electrical stimulation, and unweighted ambulation, may be used in the acute setting, but they have not been shown to be definitively efficacious over other treatment options $[1,4,6-8,10,13,30]$.

\section{Subacute phase}

Modify the training routine After the acute phase, the goal of treatment should focus on modifying training regimens and addressing biomechanical abnormalities [1, 6, $13,27]$. Decreasing weekly running distance, frequency, and intensity by $50 \%$ will likely improve symptoms without complete cessation of activity $[1,6,13,30]$. Runners are encouraged to avoid running on hills and uneven or very firm surfaces [8]. Synthetic track or a uniform surface of moderate firmness provides more shock absorption and cause less strain on the lower extremity [1] (Table 2).
Table 2 Summary of treatment goals for MTSS

Rest and ice in the acute phase

Modify training program: decrease intensity, frequency, and duration

Use low-impact and cross-training exercises during rehabilitation period

Gradually return to sport with pain-free activity

Perform regular stretching and strengthening exercises

Wear proper-fitting shoes with good shock absorption

Change shoes every 250-500 miles

Consider orthotics if indicated

Female athletes may have special considerations

Treat key dysfunctions of the entire kinetic chain; use manual therapy Consider other treatment options: ESWT, injections, acupuncture

Surgery for recalcitrant cases

During this time, athletes can benefit from cross training with other low-impact exercises, such as pool running, swimming, using an elliptical machine, or riding a stationary bicycle $[4,6,13,30]$. Clinicians can work with athletes to develop an appropriate rehabilitation plan with emphasis on proper technique, gait retraining, and return to activity in a step-wise fashion. Over a period of weeks, athletes may slowly increase training intensity and duration and add sport-specific activities, jumping exercises, and hill running to their rehabilitation program as long as they remain pain-free [1]. Athletes should scale back any exercises that exacerbate their symptoms or cause pain.

Stretching and strengthening exercises Literature has widely supported a daily regimen of calf stretching and eccentric calf exercises to prevent muscle fatigue (see Fig. 3a, b) [1, 4, 6-8, 10, 13, 27, 31, 32]. Other exercises focus on strengthening the tibialis anterior and other muscles controlling both inversion and eversion of the foot.

Patients may also benefit from strengthening core hip muscles [8, 9, 13-16, 33]. Developing core stability with strong abdominal, gluteal, and hip muscles can improve running mechanics and prevent lower-extremity overuse injuries. Developing muscle strength will improve endurance, but should not be done in the acute phase as they may exacerbate the injury due to increased strain on the tibia [1].

Footwear Many reports have found that appropriate footwear can reduce the incidence of MTSS [1, 5, 8, 13, 30]. Athletes should seek out shoes with sufficient shockabsorbing soles and insoles, as they reduce forces through the lower extremity and can prevent repeat episodes of MTSS [1, 5]. Shoes should fit properly with a stable heel counter. Some physicians recommend alternating running shoes especially when one pair is wet, as this compromises the shoe's integrity. Runners should also change running 
Fig. 3 Eccentric calf stretches and strengthening exercises.

Courtesy: R. Michael Galbraith
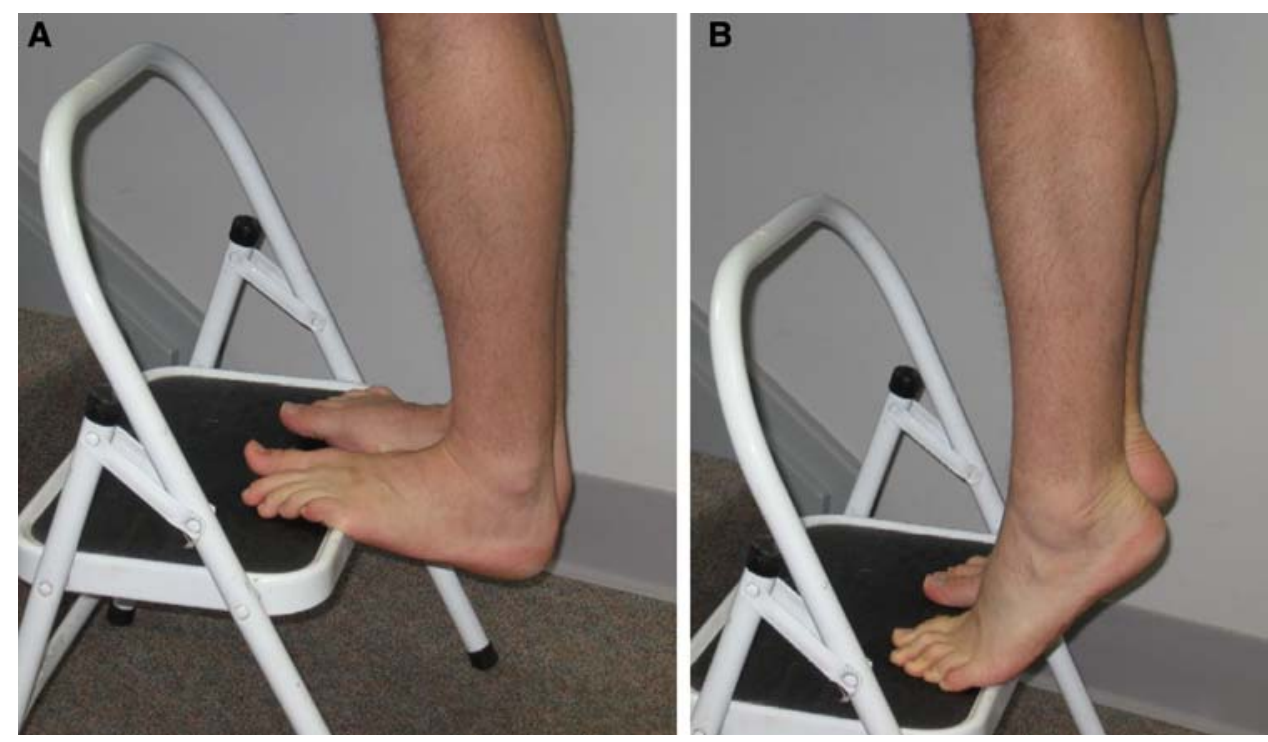

shoes every 250-500 miles, a distance at which most shoes lose up to $40 \%$ of their shock-absorbing capabilities and overall support $[1,7,34]$.

Orthotics Individuals with biomechanical problems of the foot may benefit from orthotics [4, 6, 7, 9, 13, 30]. Often, over-the-counter orthosis (flexible or semi-rigid) are sufficient to help with excessive foot pronation and pes planus. Mal-alignments caused by forefoot or rearfoot abnormalities may benefit from custom orthotics $[6,13$, 15].

Manual therapy Treatment for MTSS should include correction of key dysfunctions of the kinetic chain [8, 22, 25]. Manual therapy may be used to correct musculoskeletal abnormalities of the spine, sacroiliac joint, pelvis, and various muscle imbalances. A wide variety of manual medicine techniques, including osteopathic manipulation and physical therapy, can be used to address these dysfunctions [13]. The goal of manual medicine is to restore normal range of motion of joints, improve symmetry of muscles and soft tissues and, ultimately, restore maximal function of the body as a unit [22]. Correcting musculoskeletal dysfunctions can improve pain and overall function and may be helpful in preventing recurrence. Manual medicine has been commonly used to treat other lower extremity injuries with the benefit of improved pain and function [22, 24, 25, 35-38]. However, there is a paucity of RCTs about the role of manual medicine in treating specifically MTSS.

Proprioceptive training Proprioceptive balance training is crucial in neuromuscular education [10, 22]. This can be done with a one-legged stand, wobble board, or balance board. Improved proprioception will increase the efficiency of joint and postural-stabilizing muscles and help the body react to running surface incongruities, also key in preventing re-injury.

Intrinsic factors and gender Clinicians may need to address certain intrinsic factors with at-risk female athletes, including nutritional, hormonal, and other medical abnormalities [10]. Proper calcium replacement (ranging from 1000 to $2000 \mathrm{mg}$ daily) and Vitamin D (800 IU daily) are essential for bone strength and commonly prescribed for females [4, 13]. Estrogen supplementation (i.e., oral contraceptive pills) may be considered to help restore normal menstruation and increase bone density [1, 10]. Female athletes with the above disorders should have a thorough medical evaluation with a DEXA scan and proper psychosocial evaluation and treatment [10, 29].

Splinting/bracing Crutches may be necessary for temporary non-weightbearing and rest. Casting of the limb or a pneumatic brace is only recommended for more severe cases of MTSS and tibial stress fractures [1, 4, 6, 8, 13].

Other potential treatments Extracorporeal shock wave therapy-Extracorporeal shock wave therapy (ESWT) has been used to treat various tendinopathies of the lower extremity with varying success. Studies show mixed results with ESWT for treating tendinopathies of the foot and ankle, including plantar fasciitis and Achilles tendonitis [39-43]. No RCTs were identified for ESWT and MTSS.

Injections-Various injection methods, including cortisone, have successfully been used for decades to treat injuries of the lower extremity [18, 44-47]. Newer methods, such as dry-needling, autologous blood injection, 
platelet-rich plasma, and prolotherapy, seek to stimulate a local healing response in injured tissues. Some physicians have proposed injecting the spring and short plantar ligaments to treat laxity and poor mechanics of the foot arch, which are common factors contributing to hyperpronation [18]. However, no RCTs have been performed with these different injection techniques for MTSS.

Acupuncture-One study identified benefit of acupuncture for MTSS, but the study had a small sample size and various methodological shortcomings [48]. One case report and review article showed potential benefit with acupuncture for plantar fasciitis, but no other studies were identified for acupuncture for other lower extremity injuries [49-51].

The efficacy of ESWT, injections, and acupuncture remains unclear due to conflicting results of trials and insufficient research. As some of these potential treatments have shown benefit for numerous other lower extremity musculoskeletal conditions, one wonders if this can be extrapolated for MTSS. Clearly, further research is warranted.

\section{Surgical options}

The vast majority of individuals with MTSS have significant improvement, if not complete resolution, of their symptoms with conservative management. Surgery for MTSS is usually reserved for recalcitrant cases who do not respond with conservative treatment $[3,6,8,30]$. A "posterior fasciotomy" is the common procedure performed. This may include cauterization of the posteromedial ridge of the tibia. Surgical results are variable and not likely to cause complete resolution of symptoms but may improve pain and function [3, 15, 52, 53]. Intramedullary nail placement has been used for those with stress fractures in season.

\section{Injury prevention}

The key to treatment is prevention. Individuals with MTSS are highly susceptible to re-injury, especially if training errors, alignment abnormalities and poor technique are not corrected [1]. Physicians, athletic trainers, physical therapists, and coaches can educate athletes about proper injury prevention and develop an appropriate, individualized rehabilitation program.

\section{Conclusion}

MTSS is one of the most common lower-extremity injuries suffered by athletes and is a common presenting complaint at primary care, sports medicine, and orthopedic offices.
Prompt diagnosis and appropriate management of MTSS is important in helping athletes return to full activity in a timely fashion.

To date, there is limited evidence to support our current treatment and interventions for MTSS [5]. However, most studies support rest, ice, and analgesics in the acute phase. Many experts also recommend modifying the training routine, stretching, and strengthening the lower extremity, wearing appropriate footwear, using orthotics and manual therapy to correct biomechanical abnormalities, and gradually return to activity. ESWT, injections, and acupuncture are commonly used for other lower extremity tendinopathies with some success, but little current objective evidence exists for their role in MTSS. Ultimately, there is increased need for high-quality investigative study of conservative treatment options for this common condition, including potentially useful tools not yet thoroughly studied.

Open Access This article is distributed under the terms of the Creative Commons Attribution Noncommercial License which permits any noncommercial use, distribution, and reproduction in any medium, provided the original author(s) and source are credited.

\section{References}

1. Beck B. Tibial stress injuries: an aetiological review for the purposes of guiding management. Sports Med. 1998;26(4):265-79.

2. Anderson M, Ugalde V, Batt M, Gacayan J. Shin splints: MR appearance in a preliminary study. Radiology. 1997;204:177-80.

3. Detmer D. Chronic shin splints. Classification and management of medial tibial stress syndrome. Sports Med. 1986;3(6):436-46.

4. Fredericson M, Bergman G, Hoffman K, Dillingham M. Tibial stress reaction in runners: correlation of clinical symptoms and scintigraphy with a new magnetic resonance imaging grading system. Am J Sports Med. 1995;23:427-81.

5. Thacker S, Gilchrist J, Stroup D, Kimsey C. The prevention of shin splints in sports: a systematic review of literature. Med Sci Sports Exerc. 2002;34(1):32-40.

6. Kortebein P, Kaufman K, Basford J, Stuart M. Medial tibial stress syndrome. Med Sci Sports Exerc. 2000;32(3 suppl):S27-33.

7. Fredericson M. Common injuries in runners. Diagnosis, rehabilitation and prevention. Sports Med. 1996;21:49-72.

8. Wilder R, Seth S. Overuse injuries: tendinopathies, stress fractures, compartment syndrome, and shin splints. Clin Sports Med. 2004;23:55-81.

9. Strakowski J, Jamil T. Management of common running injuries. Phys Med Rehabil Clin N Am. 2006;17(3):537-52.

10. Dugan S, Weber K. Stress fracture and rehabilitation. Phys Med Rehabil Clin N Am. 2007;18(3):401-16.

11. Brunet M, Cook S, Brinker M, Dickson J. A survey of running injuries in 1505 competitive and recreational runners. J Sports Med Phys Fitness. 1990;30(3):307-15.

12. Yates B, White S. The incidence and risk factors in the development of medial tibial stress syndrome among naval recruits. Am J Sports Med. 2004;32(3):772-80.

13. Couture C, Karlson K. Tibial stress injuries: decisive diagnosis and treatment of 'shin splints'. Phys Sportsmed. 2002;30(6):29_ 36. 
14. Plastaras C, Rittenberg J, Rittenberg K, Press J, Akuthota V. Comprehensive functional evaluation of the injured runner. Phys Med Rehabil Clin N Am. 2005;16(3):623-49.

15. Sommer H, Vallentyne S. Effect of foot posture on the incidence of medial tibial stress syndrome. Med Sci Sports Exerc. 1995;27: $800-4$.

16. Niemuth $\mathrm{P}$, Johnson $\mathrm{R}$, Myers $\mathrm{M}$, Thieman $\mathrm{T}$. Hip muscle weakness and overuse injuries in recreational runners. Clin $\mathrm{J}$ Sport Med. 2005;15(1):14-21.

17. Messier S. Etiologic factors associated with selected running injuries. Med Sci Sports Exerc. 1998;20:501-5.

18. Ravin T, Cantieri M, Pasquarello G. Principles of prolotherapy, vol. 233. Denver, CO: American Academy of Musculoskeletal Medicine; 2008. p. 250-1.

19. Thijs Y, Van Tiggelen D, Willems T, De Clercq D, Witvrouw E. Relationship between hip strength and frontal plane posture of the knee during a forward lunge. Br J Sports Med. 2007;41:723-7.

20. Nadler F, Malanga G, DePrince M, Stitik T, Feinberg J. The relationship between lower extremity injury, low back pain, and hip muscle strength in male and female collegiate athletes. Clin J Sport Med. 2000;10(2):89-97.

21. Leetun D, Ireland M, Wilson J, Ballantyne B, Davis I. Core stability measures as risk factors for lower extremity injury in athletes. Med Sci Sports Exerc. 2004;36(6):926-34.

22. Greenman P. Principles of manual medicine. 3rd ed., chap. 11. Philadelphia, PA: Lippincott Williams \& Wilkins; 2003: p. 337403, 489.

23. Kibler W, Chandler T, Pace B. Principles of rehabilitation after chronic tendon injuries. Clin Sports Med. 1992;11(3):661-71.

24. Howell J. Effect of counterstrain on stretch reflexes, Hoffmann reflexes, and clinical outcomes in subjects with plantar fasciitis. J Am Osteopath Assoc. 2006;106(9):547-56.

25. Karageanes S. Principles of manual sports medicine. Philadelphia, PA: Lippincott, Williams and Wilkins; 2005. p. 467-8.

26. Stanitski C, McMaster J, Scranton P. On the nature of stress fractures. Am J Sports Med. 1978;6(6):391-6.

27. Korkola M, Amendola A. Exercise-induced leg pain. Sifting through a broad differential. Phys Sportsmed. 2001;29(6):35-50.

28. Mellion M, Walsh W, Madden C, Putukian M, Shelton G. The team physician's handbook. 3rd ed. Philadelphia, PA: Hanley \& Belfus; 2002. p. 517, 583.

29. Young A, McAllister D. Evaluation and treatment of tibial stress fractures. Clin Sports Med. 2006;25(1):117-28.

30. DeLee J, Drez D, Miller M. DeLee and Drez's orthopaedic sports medicine principles and practice. Philadelphia, PA: Saunders; 2003. p. 2155-9.

31. Taube R, Wadsworth L. Managing tibial stress fractures. Phys Sportsmed. 1993;21:123-30.

32. Herring K. A plyometric training model used to augment rehabilitation from tibial fasciitis. Curr Sports Med Rep. 2006;5(3): 147-54.

33. Hootman J, Macera C, Ainsworth B, Martin M, Addy C, Blair S. Predictors of lower extremity injury among recreationally active adults. Clin J Sport Med. 2002;12:99-106.

34. Cook S, Kester M, Brunet M. Shock absorption characteristics of running shoes. Am J Sports Med. 1985;13:248-53.

35. Stuber K, Kristmanson K. Conservative therapy for plantar fasciitis: a narrative review of randomized controlled trials. J Can Chiropr Assoc. 2006;50(2):118-33.
36. Pedowitz R. Use of osteopathic manipulative treatment for iliotibial band friction syndrome. J Am Osteopath Assoc. 2005; 105(12):563-7.

37. Howell J. Stretch reflex and Hoffmann reflex responses to osteopathic manipulative treatment in subjects with Achilles tendinitis. J Am Osteopath Assoc. 2006;106(9):537-45.

38. Dimou E. A randomized controlled trial (with blinded observers) of chiropractic manipulation and Achilles stretching vs. orthotics for the treatment of plantar fasciitis. J Am Chiropr Assoc. 2004;41(9):32-42.

39. Rompe J, Decking J, Schoellner C, Nafe B. Shock wave application for chronic plantar fasciitis in running athletes. A prospective, randomized, placebo-controlled trial. Am J Sports Med. 2003;31:268-75.

40. Speed C, Nichols D, Wies J, Humphreys H, Richards C, Burnet S, et al. Extracorporeal shock wave therapy for plantar fasciitis. A double blind randomised controlled trial. J Orthop Res. 2003;21: 937-40.

41. Ogden J. Extracorporeal shock wave therapy for plantar fasciitis: randomised controlled multicentre trial. Br J Sports Med. 2004; 38:382.

42. Rompe J, Nafe B, Furia J, Maffulli N. Eccentric loading, shockwave treatment, or a wait-and-see policy for tendinopathy of the main body of tendo Achilles: a randomized controlled trial. Am J Sports Med. 2007;35(3):374-83.

43. Sems A, Dimeff R, Iannotti J. Extracorporeal shock wave therapy in the treatment of chronic tendinopathies. J Am Acad Orthop Surg. 2006;14(4):195-204.

44. Hackett G, Hemwall G, Montgomery G. Ligament and tendon relaxation treated by prolotherapy. 5th ed. Oak Park, IL: Gustav A. Hemwall; 1992.

45. James S, Ali K, Pocock C, Robertson C, Walter J, Bell J. Ultrasound guided dry needling and autologous blood injection for patellar tendinosis. Br J Sports Med. 2007;41(8):518-22.

46. Hoksrud A, Öhberg L, Alfredson H, Bahr R. Ultrasound-guided sclerosis of neovessels in painful chronic patellar tendinopathy: a randomized controlled trial. Am J Sports Med. 2006;34(11): $1738-46$.

47. Öhberg L, Alfredson H. Ultrasound guided sclerosis of neovessels in painful chronic Achilles tendinosis: pilot study of a new treatment. Br J Sports Med. 2002;36(3):73-7.

48. Callison M. Acupuncture and tibial stress syndrome (shin splints). J Chinese Med. 2002;70:24-7.

49. Tillu A, Gupta S. Effect of acupuncture treatment on heel pain due to plantar fasciitis. Acupunct Med. 1998;16(2):66-8.

50. Kullman J, Steinbock K. Chinese medicine perspective... plantar fascitis. J Bodyw Mov Ther. 2001;5(1):31-3.

51. Devitt M. Electroacupuncture for plantar fasciitis: treatment reduces pain, improves function with heel pain patients. Acupunct Today. 2001;2(12):1-22.

52. Yates B, Allen M, Barnes M. Outcome of surgical treatment of medial tibial stress syndrome. J Bone Joint Surg. 2003;85:1974-80.

53. Abramowitz A, Schepsis A, McArthur C. The medial tibial syndrome: the role of surgery. Orthop Rev. 1994;23(11):875-81.

54. Willems T. Gait-related risk factors for exercise-related lower-leg pain during shod running. Med Sci Sports Exerc. 2007;39(2): 330-9.

55. Hreljac A. Impact and overuse injuries in runners. Med Sci Sports Exerc. 2004;36(5):845-9. 